\title{
Robust principal components: A generalized variance perspective
}

\author{
RAND R. WILCOX \\ University of Southern California, Los Angeles, California
}

\begin{abstract}
This article compares several methods for performing robust principal component analysis, two of which have not been considered in previous articles. The criterion here, unlike that of extant articles aimed at comparing methods, is how well a method maximizes a robust version of the generalized variance of the projected data. This is in contrast to maximizing some measure of scatter associated with the marginal distributions of the projected scores, which does not take into account the overall structure of the projected data. Included are comparisons in which distributions are not elliptically symmetric. One of the new methods simply removes outliers using a projection-type multivariate outlier detection method that has been found to perform well relative to other outlier detection methods that have been proposed. The other new method belongs to the class of projection pursuit techniques and differs from other projection pursuit methods in terms of the function it tries to maximize. The comparisons include the method derived by Maronna (2005), the spherical method derived by Locantore et al. (1999), as well as a method proposed by Hubert, Rousseeuw, and Vanden Branden (2005). From the perspective used, the method by Hubert et al. (2005), the spherical method, and one of the new methods dominate the method derived by Maronna.
\end{abstract}

\section{Introduction}

Principal components analysis (PCA) is aimed at finding a small number of $p$ linear combinations of $m$ observed variables that explain most of the variability in the data. From basic principles, the fundamental strategy focuses on the variance of the marginal distributions of the $p$ linear combinations. That is, the first component is the normalized linear combination that maximizes the variance. Then the second component is chosen from among all normalized linear combinations that are orthogonal to the first component with the property that the variance is maximized-the point being that this approach does not take into account the overall scatter of the resulting scores.

The classic technique is based on the usual covariance matrix, but it is well known that the usual covariance matrix is not robust (e.g., Hampel, Ronchetti, Rousseeuw, \& Stahel, 1986; Rousseeuw \& Leroy, 1987; Wilcox, 2005). In practical terms, situations are encountered in which, upon closer scrutiny, the resulting components explain a structure that has been created by a mere one or two outliers (e.g., Huber, 1981, p. 199). This has led to numerous suggestions regarding how the classic PCA method might be made more robust. A simple approach is to replace the covariance matrix with a robust scatter matrix.

Devlin, Gnanadesikan, and Kettenring (1981) and Campbell (1980) used an M estimate, but it has a low breakdown point, roughly meaning that a relatively small number of outliers can cause practical problems. The minimum volume ellipsoid (MVE) estimator (Rousseeuw, 1985) has a high breakdown point, but in comparison with other methods that might be used, results in Maronna (2005) suggest that this approach be abandoned. Another possibility is the fast minimum covariance determinant (MCD) estimator in Rousseeuw and Van Driessen (1999).

Results reported by Hawkins and Olive (2002) raise concerns about this estimator for reasons that go beyond PCA. (Also, the fast MCD estimator is intended as an approximation of the MCD estimator, but on the basis of results in Bernholt \& Fischer, 2004, and Bernholt, 2006, it can provide a poor approximation.) But as a diagnostic tool, MCD seems to have practical value when used in conjunction with some of the methods compared here.

A method based on $S$ estimates was studied by Croux and Haesbroeck (2000), and a fast and simple method was proposed by Locantore et al. (1999). Li and Chen (1985) suggest a "projection pursuit" approach, meaning that directions are sought that maximize or minimize some robust measure of dispersion. (One appealing feature of projection-type methods is that they can be used when the number of variables exceeds the sample size.) Croux and Ruiz-Gazen (2005, Section 5.1) describe an algorithm for implementing the $\mathrm{Li}$ and $\mathrm{Chen}$ method. (Also see Hubert, Rousseeuw, \& Verboven, 2002; Salibián-Barrera, Van Aelst, \& Willems, 2006.) One negative feature of the $\mathrm{Li}$ and Chen method is its computational complexity. Recently, Maronna (2005) extended this projection pursuit technique in a manner that improves computational efficiency and statistical performance. Yet another 
recent suggestion was made by Hubert, Rousseeuw, and Vanden Branden (2005), which was later refined by Engelen, Hubert, and Vanden Branden (2005), and is used here. Roughly, the first step is to compute a measure of "outlyingness" for each of the $n$ points, where $n$ is the sample size. Then for $h$, chosen by the investigator, the $h$ least outlying data points are used to compute a measure of location and scatter, which in turn are used to determine how many components will be retained, as well as the projected data points. Following Engelen et al., a reweighting step is added based on the orthogonal distances of the observations with respect to the first estimated PCA subspace. (So it is only at the first stage of the algorithm that the number of points eliminated is determined by the investigator.)

An interesting alternative to MCD is Olive's (2004, 2007) estimator of location and scatter (RMBA), which is based on his median ball algorithm. Olive and Hawkins (2007) show that it is consistent, and so when using this estimator in conjunction with the new methods considered here, there is hope of developing relevant theoretical results. For brevity, results using the RMBA estimator are not reported here, but it is noted that in the simulation results summarized later in the article, there seems to be little separating RMBA from MCD.

One of the new methods considered here is similar to the Hubert et al. (2005) approach, in the sense that the most outlying points are eliminated, a basic difference being that the points eliminated are those declared outliers using a method suggested by results in Wilcox (in press), and then the classic PCA method is applied to the remaining points. (Details are given in Sections 3 and 4.) The other new method is essentially a projection pursuit technique based on the goal of maximizing a robust generalized variance applied to the projected data. It is the only method aimed directly at maximizing a robust generalized variance. Here, however, an exact method for finding this maximum is not used. Rather, to keep the computational time reasonable, the (robust) generalized variances of the scores resulting from a collection of random projections are computed, and the chosen projection is the one that gives the largest result, the point being that other robust methods might yield a larger generalized variance, which is found to be the case in situations to be described.

The essential difference between this article and others aimed at comparing robust methods is the criterion used. When $p=1$, the goal of maximizing some measure of scatter applied to the projected points seems like a natural and fairly evident strategy. But when $p>1$, it seems reasonable to consider some measure of scatter that reflects the overall structure of the data, as opposed to focusing on the marginal distributions. And a seemingly natural approach is to use a robust analog of the generalized variance. This is not to suggest that methods that focus on some measure of scatter applied to the marginal distributions have no value. (And some reasons why they remain useful are discussed in the final section of this article.) But in terms of capturing the structure of the data, using a robust analog of the generalized variance would seem to be potentially more informative, particularly when dealing with distributions that are not elliptically symmetric.

\section{A Motivating Example}

To help motivate this article, data from a study are examined in which the general goal was to find good predictors of reading ability among children. The sample size is $n=81$. Here, five predictors are reduced to two linear combinations. The left panel of Figure 1 shows the scores resulting from the classic PCA (with outliers among the scores removed) and the right panel shows the scores using
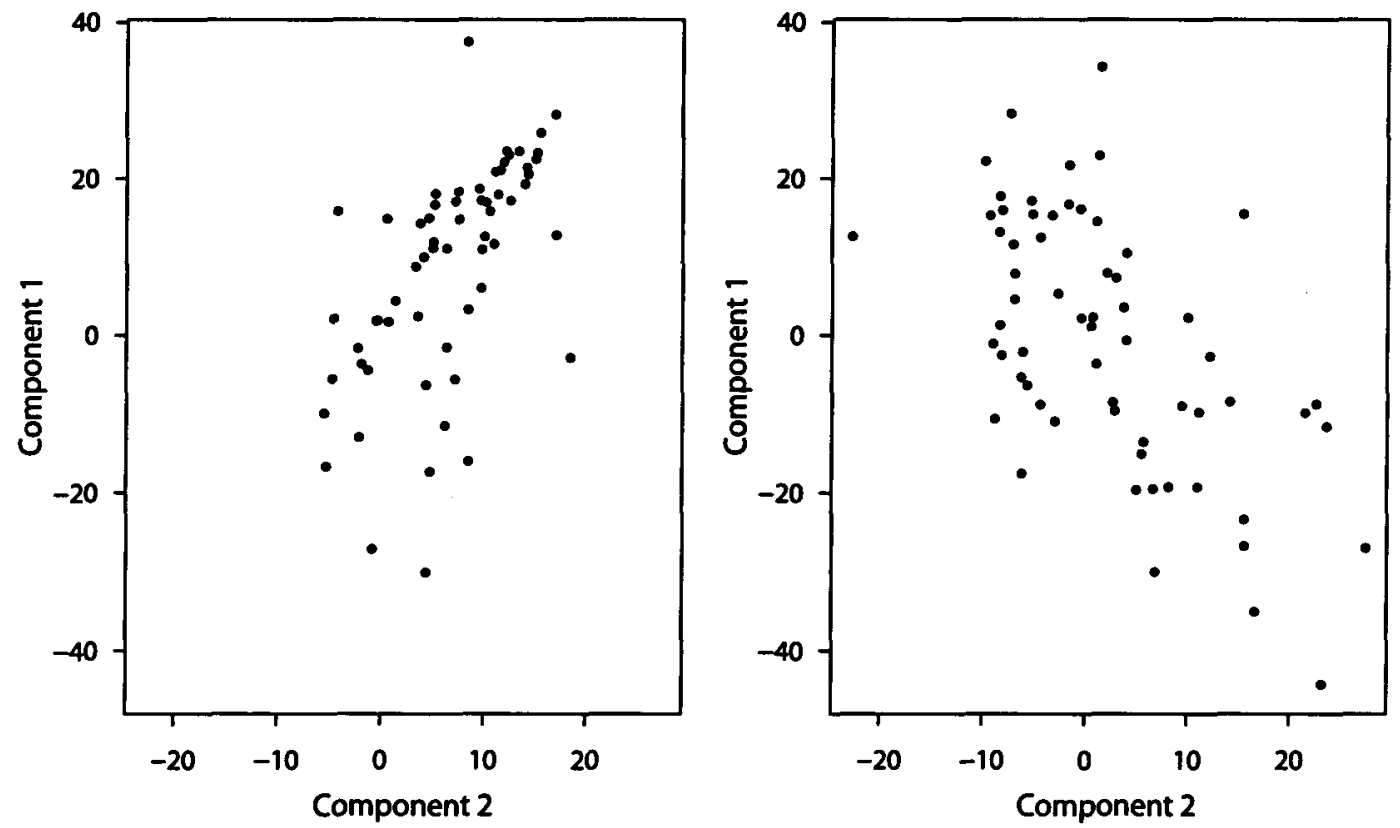

Figure 1. Scatterplot of the scores resulting from classic PCA (left panel) and method RPCA (right panel). 
method RPCA, which is described later. We see that, taken as a whole, there is more scatter among the RPCA scores. Indeed, using the robust generalized variance described at the end of Section 3, applied to all of the scores (including outliers), the generalized variance of the RPCA scores is more than six times larger than the generalized variance of the scores resulting from the classic PCA method. And if outliers among the scores are removed, the usual generalized variance of the RPCA scores is about 3.5 times larger.

An interesting aside is that the scores resulting from the classic PCA have correlation zero, as intended, but this is due to five extreme outliers. If all scores declared outliers by the projection method described in Section 3 are removed, the resulting Pearson correlation is approximately .61. The robust PCA methods considered here are not designed with the goal that the resulting components be approximately independent, but situations are encountered in which they perform substantially better in this regard. (For example, a smooth of two components will produce, approximately, a straight horizontal line, in contrast to a smooth applied to the projected data resulting from the classic PCA.)

\section{Some Background}

One of the new PCA methods considered here is based on a collection of extant techniques dealing with multivariate data in a robust manner. This section briefly reviews the methods and issues that are relevant.

Comments on detecting multivariate outliers. When choosing a multivariate outlier detection technique, at least two fundamental properties are of interest. The first is the outside rate per observation, which is the expected proportion of outliers among a sample of size $n$, say, $p_{n}$. When sampling from a multivariate normal distribution, it is generally desired to have $p_{n}$ reasonably small, say, .05, and often methods are "tuned" to achieve this goal, at least when $n$ is large (e.g., Rousseeuw \& van Zomeren, 1990). Moreover, if data are generated from a multivariate normal distribution, and if outliers are added that have an unusually large Mahalanobis distance based on the (population) mean and covariance used to generate the data, then a good multivariate outlier detection method should have a relatively high probability of flagging such points as outliers. (Another fundamental requirement is that a method should be affine equivariant. So in particular, with any change in location or rotation of the axes, points originally declared outliers would be declared outliers again.) Several methods were recently compared by Wilcox (in press), and no method dominated. But the results suggest that two methods are usually best for general use. The first uses a projection method (e.g., Wilcox, 2003, Section 13.1.9) and the other is based on a slight modification of the so-called minimum generalized variance (MGV) method (e.g., Wilcox, 2003, Section 13.1.5). The MGV method begins by finding a robust center of the data cloud, and the modification consists of using the median ball algorithm proposed by Olive (2004). The projection method, detailed in the next subsection, does not dominate the MGV method, but there is some evidence that the projection method is usually a bit better, and so it is used here. (And a technical concern about the median ball algorithm is that it is not orthogonally equivariant.)

A projection method for detecting outliers. Consider any projection of the data onto a straight line. A projection-type method for detecting outliers among multivariate data is based on the idea that if a point is an outlier, then it should be an outlier for some projection of the $n$ points. So if it were possible to consider all possible projections, and if for some projection a point is an outlier, then the point is declared an outlier. Not all projections can be considered, so following Wilcox (2005), the strategy is to orthogonally project the data onto all $n$ lines formed by the center of the data cloud, as represented by $\hat{\xi}$, and each $\mathbf{x}_{i}$. It seems natural that $\hat{\xi}$ should have a high breakdown point, and two were considered here. Following Croux and Ruiz-Gazen (2005), as well as Hubert et al. (2002), the first choice for $\xi$ was taken to be the $L_{1}$ median (sometimes called the spatial median), which has a breakdown point of .5 , the highest possible value (e.g., Rousseeuw \& Leroy, 1987, p. 251). That is, $\hat{\xi}$ is taken to be the value that minimizes

$$
\Sigma\left\|\hat{\xi}-\mathbf{x}_{i}\right\|,
$$

where

$$
\mathbf{x}_{i}=\sqrt{x_{1}^{2}+\cdots+x_{p}^{2}}
$$

is the usual Euclidean norm. Here, $\hat{\xi}$ was computed with the $R$ function spat in Wilcox $(2005$, p. 238). (An alternative $R$ function, called Llmedian, can be downloaded from Christophe Croux's Web page. For results on computing the $L_{1}$ median, see Bedall \& Zimmermann, 1979; Vardi \& Zhang, 2000.)

It is noted that Massé and Plante (2003) compared several robust multivariate estimators of location and concluded that the $L_{1}$ median performed best. Here, consideration was also given to the fast MCD estimator. In simulations, the choice between these two measures of location generally made a trivial difference. But when dealing with skewed, heavy-tailed distributions, there were rare instances in which MCD gave better results. Here, for brevity, only results using fast MCD are reported. It is noted that consideration was also given to the marginal medians. In terms of the simulation results in Section 5, the measure of location used seems to make a minor difference.

Given $\xi$, the remaining computational details of the projection method for detecting outliers are as follows. Fix $i$, and for the point $\mathbf{x}_{i}$, orthogonally project all $n$ points onto the line connecting $\hat{\xi}$ and $x_{i}$, and let $D_{i j}$ be the distance between $\hat{\xi}$ and $x_{j}$ based on this projection. More formally, let

$$
\begin{aligned}
& \mathbf{A}_{i}=x_{i}-\hat{\xi}, \\
& \mathbf{B}_{j}=x_{j}-\xi,
\end{aligned}
$$

where both $\mathbf{A}_{i}$ and $\mathbf{B}_{j}$ are column vectors having length $p$, and let

$$
\mathbf{C}_{j}=\frac{\mathbf{A}_{i}^{\prime}}{\mathbf{B}_{j}^{\prime}} \frac{\mathbf{B}_{j}}{\mathbf{B}_{j}} \mathbf{B}_{j}
$$


$j=1, \ldots, n$. Then, when projecting the points onto the line between $x_{i}$ and $\hat{\xi}$, the distance of the $j$ th point from $\xi$ is

$$
D_{i j}=\left\|\mathbf{C}_{j}\right\| \text {. }
$$

Here, an extension of Carling's (2000) modification of the boxplot rule is used to check for outliers among the $D_{i j}$ values. (For a similar boxplot approach, see Brys, Hubert, \& Rousseeuw, 2005.) Let $\ell=[n / 4+5 / 12]$, where [.] is the greatest integer function, and let

$$
h=\frac{n}{4}+\frac{5}{12} \text {. }
$$

For fixed $i$, let $D_{i(1)} \leq \ldots \leq D_{i(n)}$ be the $n$ distances written in ascending order. The ideal fourths associated with the $D_{i j}$ values are

$$
q_{1}=(1-h) D_{i(h)}+h D_{i(h+1)}
$$

and

$$
q_{2}=(1-h) D_{i(\ell)}+h D_{i(\ell-1)} \text {. }
$$

Then the $j$ th point is declared an outlier if

$$
D_{i j}=M_{D}+\sqrt{\chi_{.975, p}^{2}}\left(q_{2}-q_{1}\right),
$$

where $M_{D}$ is the usual sample median based on the $D_{i 1}, \ldots$, $D_{\text {in }}$ values.

The process just described is for a single projection; for fixed $i$, points are projected onto the line connecting $\mathbf{x}_{i}$ to $\xi$. Repeating this process for each $i, i=1, \ldots, n$, a point is declared an outlier if for any of these projections, it satisfies Equation 1. (The R function outpro in Wilcox, 2005, applies this method and was used here.) This approach has certain similarities with a projection method suggested by Peña and Prieto (2001). One important difference is that the method used by Peña and Prieto is based on the usual sample mean, which is not robust and which, in turn, could result in masking.

For completeness, it is noted that Hubert et al. (2005) use MCD on projected data to search for outliers. One reason their method seems interesting is that it provides details about different types of outliers that might occur.

Note that a general way of computing a robust generalized variance is to use the determinant of some robust covariance matrix. One way of computing a robust covariance matrix is simply to remove any points flagged as outliers by the projection method just described and compute the usual covariance matrix using the remaining data, and this strategy is used here unless stated otherwise. It is noted that under normality, the resulting correlations tend to be relatively close to Pearson's correlation (Wilcox, 2005). But this is not to suggest that all other robust measures of scatter be excluded from consideration.

\section{Description of the Robust PCA Methods}

This section describes the robust PCA methods that are to be compared.

Maronna's method. The method proposed by Maronna (2005) is based in part on an iterative algorithm. I will not give a complete description of the long and tedious details here, but a brief outline of the method is helpful. Let $x_{i}, i=1, \ldots, n$, be an $m$-dimensional data set, let $q=m-p$, and let $C$ be an orthonormal $q$-by- $m$ matrix. That is, $\mathbf{C C}^{\prime}=\mathbf{I}_{q}$. For some $q$-vector $\mathbf{a}$, let

$$
r_{i}(\mathbf{C}, \mathbf{a})=\|\mathbf{C x}-\mathbf{a}\|,
$$

and let $\sigma(r)$ be a scale statistic. The goal is to determine $\mathrm{C}$ and a so as to minimize $\sigma(r)$. Maronna considers two choices for $\sigma(r)$ : an $M$-scale and an $L$-scale. Here the focus is on the $L$-scale

$$
\sigma(r)=\sum_{i=1}^{h} r_{(i)},
$$

where $r_{(1)} \leq \ldots \leq r_{(h)}, h<n$, primarily because it is faster and easier to compute. Following Maronna (2005), $h$ is taken to be the largest integer less than or equal to $(n+$ $m-q+2) / 2$, where $q=m-p$. (Simulations were run with the $M$-scale, but this did not alter any of the general conclusions outlined in Section 5.) Henceforth, this will be called method M. (An R function for applying this method, called marpca, is available from the author upon request.)

The SPCA method. The spherical PCA (SPCA) procedure was derived by Locantore et al. (1999). Let $\mu$ be the $L_{1}$ median, which again was computed with the $\mathrm{R}$ function spat described in Wilcox (2005, p. 238). Let $y_{i}=\left(x_{i}-\mu\right) /$ $\left\|\mathrm{x}_{i}-\mu\right\|$. The procedure consists of using the eigenvectors $b_{1}, \ldots, b_{m}$ of the covariance matrix of the $y_{i}$. But the eigenvalues are in general not consistent, in which case they are replaced by

$$
\lambda_{j}=S\left(\mathbf{b}_{j} \mathbf{x}_{1}, \ldots, \mathbf{b}_{j} \mathbf{x}_{n}\right)^{2},
$$

where $S$ is any robust measure of scale. Following Maronna (2005), $S$ is taken to be the median absolute deviation (MAD) statistic. (For the values $X_{1}, \ldots, X_{n}, \mathrm{MAD}$ is the median of $\left|X_{1}-M\right|, \ldots,\left|X_{n}-M\right|$, where $M$ is the usual median of $X_{1}, \ldots, X_{n}$.)

Method HRVB. Hubert et al. (2005) suggest a method that combines projection pursuit ideas with robust scatter matrix estimation. And as previously indicated, an adaptation of this method was derived by Engelen et al. (2005), which is used here. The computational details are quite involved, and so only a brief outline of the method is provided.

The method begins by finding the $h$ least outlying data points. The choice for $h$ is made by the investigator, and Hubert et al. (2005) consider choices of the form $h=$ $\max \left\{[a n],\left[\left(n+k_{\max }+1\right) / 2\right]\right\}$, where $a$ is some value between .5 and 1 and $k_{\max }$ is the maximum number of components that will be computed; they use $\alpha=.75$ and $k_{\max }=$ 10 , and the same is done here. Next, outlyingness is measured using a maximum standardized distance among the class of all possible projections of the data onto a unidimensional space. Not all projections can be considered, so for $n$ small, they focus on all directions through two points, and for $n !>250(n-2) ! 2 !$, they take at random 250 projections. They then focus on the mean and covariance matrix of the $h$ points that have the smallest distances just computed. The next step computes fast MCD for the projected data resulting from the previous step, which is used to compute a reweighted mean and covariance matrix that increases statistical efficiency. A consistency factor is used to make the 
estimator unbiased at normal distributions. (An $\mathrm{R}$ function for applying this method, called robpca.SSP, is available at www.wis.kuleuven.ac.be/stat/robust.html and was used here.)

Method OP. Method OP simply removes any outliers detected by the projection approach described. Then the classic PCA is applied to the data that remain, and the $p$-dimensional representation of the data is computed in the usual way. So although the use of this projection method is not new in the context of detecting outliers, it seems that there are no results on how it performs in the context of PCA.

For completeness, it is noted that Croux and Ruiz-Gazen (2005) suggest an algorithm that begins with projections based in part on the $L_{1}$ median, but it is evident that their approach differs from method OP. Method OP attempts to eliminate outliers in a manner that takes into account the overall structure of the data. The algorithm used by Croux and Ruiz-Gazen (2005) does not do this, but rather searches for projections that maximize a robust measure of scatter applied to the marginal distributions of the scores.

Also, Croux, Filzmoser, and Oliveira (2007, pp. 6-7) note that the Croux and Ruiz-Gazen (1996) and Hubert et al. (2002) projection algorithms suffer from severe downward bias. It is unknown whether method OP suffers from the same problem.

Method RPCA. Let B be any $p$-by- $m$ orthonormal matrix. Given $\mathbf{B}$, the resulting $p$-dimensional representation of the data is

$$
\mathbf{z}_{i}=\mathbf{B} \mathbf{x}_{i}-\theta,
$$

where $\theta$ is some measure of location. (More generally, all of the methods considered here use Equation 2, and differ in how they determine $B$ and $\theta$.) Let $\hat{\Xi}$ be an estimate of some robust generalized variance based on the $z_{i}$ values. (So $\hat{\Xi}$ is the determinant of some robust covariance matrix that is computed with the $\mathbf{z}_{i}$ values.) As previously indicated, $\hat{\Xi}$ is taken to be the determinant of the covariance matrix described at the end of Section 3. As for estimating $\theta$, choices that were considered included fast MCD, the $L_{1}$ median, Olive's (2004) estimator based on the socalled median ball algorithm, and the mean of the data after points flagged as outliers by the projection method are removed. In terms of the simulation results reported here, the choice seems to make little difference, and so attention is focused on MCD.

The ideal goal is to find the B that maximizes $\hat{\Xi}$, but this goal is not necessarily achieved here because a random search is used, which in general does not find the optimal $B$. That is, randomly generate B, compute $\hat{\Xi}$, repeat this, say, $I$ times, and use the $B$ that yields the largest value for $\hat{E}$. Here, $I=100$ is used. Some experiments suggested that little is gained using $I=200$ or 300 . Of course, there might be situations in which $I>100$ gives more satisfactory results, but this increases the execution time in the simulations reported here, which are high even with $I=100$. (The R function Rpca, available from the author, applies this method.)

To elaborate slightly, random generation of B was accomplished as follows. First, generate pm random numbers from a uniform distribution and store the results in a $p$-by-m matrix. Next, the $\mathrm{QR}$ decomposition of this matrix is computed (using the $R$ function $q r$ ), which in turn can be used to compute an orthonormal matrix (using the $R$ function qr.Q).

The original goal was to use method RPCA as a benchmark for the other robust methods previously described. An initial concern about using it in practice was its random component. But given the goal of maximizing a robust generalized variance of the projected points, results reported here suggest that it has practical value. However, even though RPCA is the only method that reflects a direct attempt to maximize a robust generalized variance, situations arise in which an alternative method is more satisfactory, as will be seen.

\section{Simulation Results}

Simulations were used to compare the small-sample properties of the methods described in the previous section. Data were generated from a multivariate distribution, to be described, with $m=6$ and 10 , and $n=100$. The variables were taken to have a common correlation of $\rho=0$ or .5. Then each method was applied, resulting in a matrix $B$ of $p=4$ directions, the resulting scores were determined using Equation 2, and the generalized variance of these scores was computed. Let $\hat{\Xi}_{j}(j=1, \ldots, 6)$ be the estimate of $\Xi$ using the $j$ th method, which are classic PCA, M (Maronna), HRVB, OP, SPCA, and RPCA, respectively. Repeating this process $K$ times yields $K$ values for $\hat{\Xi}_{j}$. For the $j$ th method, let $\bar{\tau}_{j}$ be the average value of $\hat{\Xi}_{j}$ over these $K$ replications. Due to very high execution time, $K=500$ was used. Then the relative merits of the methods were measured with $\omega_{j}=\bar{\tau}_{j} / \bar{\tau}_{1}(j=2, \ldots, 6)$.

Here, the marginal distributions were taken from the class of $g$-and- $h$ distributions (Hoaglin, 1985), which includes a standard normal distribution as a special case. If $Z$ is a standard normal random variable, then

$$
X=\left(\frac{\exp (g Z)-1}{g}\right) \exp \left(h Z^{2} / 2\right)
$$

has a $g$-and- $h$ distribution. When $g=0$, this last expression is taken to be

$$
X=Z \exp \left(h Z^{2} / 2\right)
$$

The case $g=h=0$ corresponds to a standard normal distribution. When $g=0$, the distribution is symmetric, and the tails of the distribution get heavier as $h$ gets large.

Table 1 summarizes some properties of the four $g$-and- $h$ distributions used here, where skewness is measured with $\kappa_{1}=\mu_{3} / \mu_{2}^{1.5}$, and kurtosis with $\kappa_{2}=\mu_{4} / \mu_{2}^{2}$, where $\mu_{k}=$ $E(X-\mu)^{k}$. If $h>1 / k, \mu_{k}$ is not defined, meaning that in theory, the $k$ th moment is infinitely large, which is why some entries in Table 1 are blank. (Of course, on a computer, kurtosis is large but finite, because the values generated on a computer are bounded.) With $h=.5$, which is considered here, the $g$-and- $h$ distribution has extremely heavy tails, resulting in many outliers in a given sample. (Many other properties are described in Hoaglin's article.)

As for generating dependent observations, let $\mathbf{R}$ be a correlation matrix and form the Cholesky decomposition $\mathbf{U}^{\prime} \mathbf{U}=\mathbf{R}$, where $\mathbf{U}$ is the matrix of factor loadings of the 
Table 1

Some Properties of the $g$-and- $h$ Distribution

\begin{tabular}{cccc}
\hline$g$ & $h$ & $x_{1}$ & $\kappa_{2}$ \\
\hline 0 & 0 & 0.00 & 3.0 \\
0 & .5 & 0.00 & - \\
.5 & 0 & 1.75 & - \\
.5 & .5 & - & - \\
\hline
\end{tabular}

principal components of the square-root method of factoring a correlation matrix, and $\mathbf{U}^{\prime}$ is the transpose of $\mathbf{U}$. Then $\mathbf{X U}$ produces an $n$-by- $m$ matrix of data that has population correlation matrix $\mathbf{R}$.

It might help to note that all of the methods considered here are scale equivariant. That is, if each observation is multiplied by some constant $c$, the relative performance of the methods, based on the robust generalized variance used here, remains the same in the terms of $\omega_{j}$. This is because each of the robust generalized variances is multiplied by $c^{2 p}$.

The estimates of $\omega_{j}$ are shown in Table 2 . Note that under normality, there is some indication that the classic PCA method is not always optimal, although the advantage of the alternative methods is small. But when using the usual covariance matrix, now the classic PCA method is always optimal, a result that is elaborated on at the end of this section. Also, under normality, there is little separating classic PCA from the five robust alternatives considered when $m=6$. That is, any apparent advantage would appear to be trivial at best. However, when $m=10$, HRVB, OP, and SPCA seem $a$ bit better than methods $M$ and RPCA. When the marginal distributions are skewed and relatively light-tailed $(g=.5$, $h=0$ ), generally, the robust methods offer a bit of an advantage, with the possible exception of method $\mathrm{M}$ when $m=10$. When the marginal distributions have relatively heavy-tailed distributions $(h=.5)$, the robust methods generally offer a substantial improvement, with method $M$ being the least satisfactory. Method RPCA can provide the most striking improvement, but it seems that under normality, it can be the least satisfactory technique. For this reason, methods HRVB, OP, and SPCA might be deemed best for routine use. Forced to choose one method for general use, SPCA appears to be a good candidate, and it has the additional appeal of computational simplicity and relatively low execution time.

However, Hubert et al. (2005) show that SPCA is sometimes less robust than HRVB. Moreover, although computationally complex, the R software used to apply HRVB is easy to use and has relatively low execution time.

Another possibility is to consider all five methods and choose the one that yields the highest ratio. But a practical consideration might be that Maronna's method can have relatively high execution time; it was much higher than for methods HRVB, SPCA, and OP. For example, on a (relatively slow) Sun computer running Unix, with $n=$ $30, m=6$, and $p=4$, Maronna's method required $2 \mathrm{~min}$ $20 \mathrm{sec}$, versus $33 \mathrm{sec}$ for method RPCA and less than $2 \mathrm{sec}$ using the other techniques. Also, increasing $n$ was found to have a much bigger impact on execution time when using Maronna's method versus all of the other techniques. In fairness, on faster computers currently available, perhaps this issue can be ignored. However, all indications are that RPCA, HRVB, SPCA, and OP dominate Maronna's method based on the criterion used, suggesting that in terms of maximizing the robust generalized variance of the scores, Maronna's method might be eliminated.

To gain perspective, additional simulations were run using the usual generalized variance applied to the resulting scores. Under normality, classic PCA offers an advantage, as would be expected, but the improvement is not substantial. The ratios $\left(\omega_{j}\right)$ now range between .85 and .98 , with method $\mathrm{M}$ having the lowest ratio and method OP having the highest. With heavy-tailed distributions $(h=.5)$, all of the ratios drop below .5 (except for method RPCA). But again, the cost of using classic PCA is that outliers can result in scores that poorly reflect the structure of the bulk of the data.

\section{Concluding Remarks}

Although classic PCA is not robust, in some situations it competes well with the robust methods considered. For example, Williams et al. (2005) conducted a study dealing generally with the Porteus Maze Test (PMT), which is used to evaluate intelligence and executive functioning and screen for intellectual deficiency. A portion of the study dealt with the association between the so-called $\mathrm{Q}$ score resulting from the PMT test and several predictors. Three of these predictors (having to do with measures of delinquency, aggressiveness, and externalizing) were collapsed to two variables using the methods considered here. The estimated robust generalized variances ranged between 58 and 66, with classic PCA giving the largest value. Reducing the three predictors to one variable, the estimates ranged between 46 and 49, with RPCA giving the best results.

The illustration in Section 2 demonstrates that the improvement of RPCA over classic PCA can be even more striking than the improvements shown in Table 2 . It is noted that for the data used to create Figure 1, HRVB, $O P$, and SPCA also provide substantial improvement. The ratio of the robust generalized variance of the classic PCA scores, divided by the robust generalized variance of the scores resulting from HRVB, was 6.36. For methods OP and SPCA, this ratio was 6.92 and 6.84 , respectively.

Note that when $p=m$, method RPCA becomes trivial: The robust generalized variance is invariant under any rotation of the axes. So, to the extent it is desired to rotate the axes so that the resulting components have zero correlation, RPCA does not provide a solution.

Table 2

Estimates of $\omega_{j}$

\begin{tabular}{cccccccc}
\hline$g$ & $h$ & $m$ & $\begin{array}{c}\omega_{2} \\
(\mathrm{M})\end{array}$ & $\begin{array}{c}\omega_{3} \\
\text { (HRVB) }\end{array}$ & $\begin{array}{c}\omega_{4} \\
(\mathrm{OP})\end{array}$ & $\begin{array}{c}\omega_{5} \\
\text { (SPCA) }\end{array}$ & $\begin{array}{c}\omega_{6} \\
\text { (RPCA) }\end{array}$ \\
\hline 0 & 0 & 6 & 0.93 & 1.10 & 1.02 & 1.03 & 1.13 \\
0 & .5 & 6 & 1.13 & 1.75 & 1.73 & 1.74 & 2.48 \\
.5 & 0 & 6 & 1.01 & 1.30 & 1.13 & 1.27 & 1.45 \\
.5 & .5 & 6 & 1.53 & 2.22 & 1.97 & 2.11 & 3.56 \\
0 & 0 & 10 & 0.78 & 1.03 & 1.01 & 1.04 & 0.72 \\
0 & .5 & 10 & 1.59 & 3.37 & 2.59 & 3.99 & 3.77 \\
.5 & 0 & 10 & 0.86 & 1.31 & 1.19 & 1.48 & 1.14 \\
.5 & .5 & 10 & 1.82 & 3.44 & 3.03 & 5.52 & 5.23 \\
\hline
\end{tabular}


It is not being suggested that methods aimed at maximizing the variance of the marginal distributions of component scores have no practical value. In regression, for example, when dealing with multicollinearity, maximizing this variance can be beneficial in terms of power when testing hypotheses about the individual slopes. Nevertheless, when trying to reveal the structure of data, some type of generalized variance criterion would seem to be useful.

Finally, it is observed that in their simulations comparing methods, Maronna (2005), as well as Engelen et al. (2005), focus on normal distributions that have been contaminated in some manner. It appears that there are no results on how methods compare when distributions are not elliptically symmetric. This article has offered one possible approach regarding how this might be done.

\section{AUTHOR NOTE}

Correspondence concerning this article should be addressed to R. R. Wilcox, Department of Psychology, University of Southern California, Los Angeles, CA 90089-1061 (e-mail: rwilcox@usc.edu).

\section{REFERENCES}

Bedall, F. K., \& ZimmermanN, H. (1979). Algorithm AS 143: The mediancentre. Applied Statistics, 28, 325-328.

BERNHOLT, T. (2006). Robust estimators are hard to compute (Technical report). 1s2-www.cs.uni-dortmund.de/ bernholt/ps/tr52-05.pdf.

BERNHOLT, T., \& Fischer, P. (2004). The complexity of computing the MCD estimator. Theoretical Computer Science, 326, 383-393.

BRYS, G., HubERT, M., \& RousseeUW, P. J. (2005). A robustification of independent component analysis. Journal of Chemometrics, 19, 364-375.

CampBell, N. A. (1980). Robust procedures in multivariate analysis I: Robust covariance estimation. Applied Statistics, 29, 231-237.

CARLING, K. (2000). Resistant outlier rules and the non-Gaussian case. Computational Statistics \& Data Analysis, 33, 249-258.

Croux, C., Filzmoser, P., \& OliveirA, M. R. (2007). Algorithms for projection-pursuit robust principal component analysis. Chemometrics \& Intelligent Laboratory Systems, 87, 218-225.

Croux, C., \& Haesbroeck, G. (2000). Principal component analysis based on robust estimators of the covariance or correlation matrix: Influence functions and efficiencies. Biometrika, 87, 603-618.

Croux, C., \& Ruiz-Gazen, A. (1996). A fast algorithm for robust principal components based on projection pursuit. In A. Prat (Ed.), Compstat: Proceedings in computational statistics (pp. 211-216). Heidelberg: Physica.

Croux, C., \& Ruiz-Gazen, A. (2005). High breakdown estimators for principal components: The projection-pursuit approach revisited. Journal of Multivariate Analysis, 95, 206-226.

Devlin, S. J., Gnanadesikan, R., \& KetTenRing, J. R. (1981). Robust estimation of dispersion matrices and principal components. Journal of the American Statistical Association, 76, 354-362.

ENGELEN, S., HuberT, M., \& VANDEN BRANDEN, K. (2005). A comparison of three procedures for robust PCA in high dimensions. Austrian Journal of Statistics, 34, 117-126.

Hampel, F. R., Ronchetti, E. M., Rousseeuw, P. J., \& Stahel, W. A. (1986). Robust statistics: The approach based on influence functions. New York: Wiley.

HAwkINs, D. M., \& OLIVE, D. J. (2002). Inconsistency of resampling algorithms for high-breakdown regression estimators and a new algorithm. Journal of the American Statistical Association, 97, 136-159.
Hoaguin, D. C. (1985). Summarizing shape numerically: The $g$-and- $h$ distributions. In D. C. Hoaglin, F. Mosteller, \& J. W. Tukey (Eds.), Exploring data tables, trends, and shapes (pp. 461-514). New York: Wiley.

HUBER, P. J. (1981). Robust statistics. New York: Wiley.

Hubert, M., Rousseeuw, P. J., \& VANDEN BRanden, K. (2005). ROBPCA: A new approach to robust principal component analysis. Technometrics, 47, 64-79.

Hubert, M., RousseeUw, P. J., \& Verboven, S. (2002). A fast method for robust principal components with applications to chemometrics. Chemometrics \& Intelligent Laboratory Systems, 60, 101-111.

L1, G., \& ChEN, Z. (1985). Projection-pursuit approach to robust dispersion matrices and principal components: Primary theory and Monte Carlo. Journal of the American Statistical Association, 80, 759-766.

Locantore, N., Marron, J. S., Simpson, D. G., Tripoli, N., Zhang, J. T., \& CoHEN, K. L. (1999). Robust principal components for functional data. Test, 8, 1-28.

MaronNa, R. A. (2005). Principal components and orthogonal regression based on robust scales. Technometrics, 47, 264-273.

Massé, J.-C., \& Plante, J.-F. (2003). A Monte Carlo study of the accuracy and robustness of ten bivariate location estimators. Computational Statistics \& Data Analysis, 42, 1-26.

OLIVE, D. J. (2004). A resistant estimator of multivariate location and dispersion. Computational Statistics \& Data Analysis, 46, 93-102.

OLIVE, D. J. (2007). Applied robust statistics. Unpublished manuscript. www.math.siu.edu/olive/ol-bookp.htm.

OLIVE, D. J., \& HAWKINS, D. M. (2007). Robustifying robust estimators. Preprint (www.math.siu.edu/olive/preprints.htm).

PeÑA, D., \& PrIETo, F. J. (2001). Multivariate outlier detection and robust covariance matrix estimation. Technometrics, 43, 286-299.

RousSEEUW, P. J. (1985). Multivariate estimation with high breakdown point. In W. Grossmann, G. Pflug, \& W. Wertz (Eds.), Mathematical statistics and applications, $B$ (pp. 283-297). Dordrecht: Reidel.

ROUSSEEUW, P. J., \& LEROY, A. M. (1987). Robust regression and outlier detection. New York: Wiley.

Rousseeuw, P. J., \& VAN Driessen, K. (1999). A fast algorithm for the minimum covariance determinant estimator. Technometrics, 41, 212-223.

RousseeuW, P. J., \& VAN ZOMEREN, B. C. (1990). Unmasking multivariate outliers and leverage points. Journal of the American Statistical Association, 85, 633-639.

Salibián-Barrera, M., Van Aelst, S., \& Willems, G. (2006). Principal components analysis based on multivariate $M M$ estimators with fast and robust bootstrap. Journal of the American Statistical Association, 101, 1198-1211.

VARDI, Y., \& ZHANG, C.-H. (2000). The multivariate $L_{1}$-median and associated data depth. Proceedings of the National Academy of Sciences, 97, 1423-1426.

Wincox, R. R. (2003). Applying contemporary statistical techniques. New York: Academic Press.

WrLcox, R. R. (2005). Introduction to robust estimation and hypothesis testing (2nd ed.). New York: Academic Press.

WrLcox, R. R. (in press). Some small-sample properties of some recently proposed multivariate outlier detection techniques. Journal of Statistical Computation \& Simulation.

Williams, N., Stanchina, J., Bezdian, S., Skrox, E., Raine, A., \& BAKER, L. (2005). Porteus' mazes and executive function in children: Standardized administration and scoring, and relationships to childhood aggression and delinquency. Unpublished manuscript. Los Angeles: University of Southern California, Department of Psychology.

(Manuscript received February 7, 2007; revision accepted for publication July 14,2007 .) 